\title{
Customizations and Expression Breakdowns in Ecosystems of Communication Apps
}

CARLA F. GRIGGIO, LRI, Univ. Paris-Sud, CNRS, Inria, Université Paris-Saclay, France and The University of Tokyo, Japan

JOANNA MCGRENERE, Dept of Computer Science University of British Columbia, Canada and LRI, Univ. Paris-Sud, CNRS, Inria, Université Paris-Saclay, France

WENDY E. MACKAY, LRI, Univ. Paris-Sud, CNRS, Inria, Université Paris-Saclay, France

The growing adoption of emojis, stickers and GIFs suggests a corresponding demand for rich, personalized expression in messaging apps. Some people customize apps to enable more personal forms of expression, yet we know little about how such customizations shape everyday communication. Since people increasingly communicate via multiple apps side-by-side, we are also interested in how customizing one app influences communication via other apps. We created a taxonomy of customization options based on interviews with 15 "extreme users" of communication apps. We found that participants tailored their apps to express their identities, organizational culture, and intimate bonds with others. They also experienced expression breakdowns: frustrations around barriers to transferring personal forms of expression across apps, which inspired inventive workarounds to maintain cross-app habits of expression, such as briefly switching apps to generate and export content for a particular conversation. We conclude with implications for personalized expression in ecosystems of communication apps.

CCS Concepts: • Human-centered computing $\rightarrow$ Empirical studies in collaborative and social computing.

Additional Key Words and Phrases: Computer-Mediated Communication (CMC); Ecosystems of Communication Apps; Expression Breakdowns; Close Relationships; Co-adaptation; Customization

ACM Reference Format:

Carla F. Griggio, Joanna McGrenere, and Wendy E. Mackay. 2019. Customizations and Expression Breakdowns in Ecosystems of Communication Apps. Proc. ACM Hum.-Comput. Interact. 3, CSCW, Article 26 (November 2019), 26 pages. https://doi.org/10.1145/3359128

\section{INTRODUCTION}

People increasingly adopt emojis, stickers and GIFs as means of expression that help them enrich and personalize their text-based communication. For example, emojis not only express emotions, but also help convey identity traits and friendly impressions for maintaining positive relationships with others [52]. People carefully select stickers that reflect their personalities during conversations [67], and also send animated GIFs based on TV shows or movies that let them react to messages with richer gestures and facial expressions than would be possible with text, emojis or stickers [2]. We believe that such practices point to the need for strong personalization in mediated communication, and are particularly interested in how technology can support more individualized, personal forms of expression that go beyond the default options offered by today's communication apps.

Authors' addresses: Carla F. Griggio, carla@iis-lab.org, LRI, Univ. Paris-Sud, CNRS, Inria, Université Paris-Saclay, F-91400, Orsay, France, The University of Tokyo, Japan; Joanna McGrenere, joanna@cs.ubc.ca, Dept of Computer Science, University of British Columbia, Vancouver, Canada , LRI, Univ. Paris-Sud, CNRS, Inria, Université Paris-Saclay, F-91400, Orsay, France; Wendy E. Mackay, mackay@lri.fr, LRI, Univ. Paris-Sud, CNRS, Inria, Université Paris-Saclay, F-91400, Orsay, France.

(C) 2019 Association for Computing Machinery.

This is the author's version of the work. It is posted here for your personal use. Not for redistribution. The definitive Version of Record was published in Proceedings of the ACM on Human-Computer Interaction, https://doi.org/10.1145/3359128. 
Although most communication apps offer customization options for tailoring notifications, user-interface aesthetics, and means of expression, we know little about their role in personal communication. For example, Facebook Messenger (henceforth Messenger) lets conversation partners change the color of their chat bubbles and set a shortcut to a frequently used emoji. Telegram lets users import custom-made stickers and install bots or chatbots [28] that automate tasks via conversational interfaces, e.g., creating a poll in a group chat to make a collective decision. Slack supports importing custom-made emojis that can then be used as reactions ${ }^{1}$. We are interested in characterizing how users leverage these and other customization options with the goal of informing novel designs for increased personalization of mobile communication.

Not only do people communicate extensively via communication apps, but they increasingly adopt a mix of different communication apps to support their everyday communication [14, 49]. For example, a user may use WhatsApp for text messaging, as well as Messenger and Telegram. Those who enjoy sharing stories [35] may use Snapchat alongside Instagram. Others use specific apps to support group work within a team, such as Slack and HipChat. Together, each user's collection of communication apps provides diverse means of expression and opportunities for showing care [9], and support sharing different types of content with different audiences [48]. In line with recent work that advocates for an ecological approach to studying mediated communication $[12,41,48,66]$, we are interested in the value that users place on the diverse customization options available across their personal ecosystems of communication apps.

This paper investigates customization practices in the context of computer-mediated communication (CMC) guided by the following research questions: 1) What customizations do people make to their communication apps, and how can we characterize them so as to inform design? 2) What value do people place on the diverse customization options available throughout their personal ecosystems of communication apps? We address these questions with an interview study of 15 "extreme users" of communication apps in order to gather rich insights into potential new designs. We contribute a taxonomy of customization options in mobile communication apps, and novel insights about the role that customizations can play in maintaining online relationships as well as the breakdowns and workarounds that arise in their attempts to proactively personalize their communication. Last, we discuss the implications of our research and show how our taxonomy creates a design space for exploring new opportunities for design.

\section{RELATED WORK}

Software customization occurs in the context of a co-adaptative process, where "people both adapt to technology (reacting to it) and adapt it to meet their needs (proactively changing it)" [31]. Mackay proposes that users adapt to technology as they learn how to work within its capabilities and limitations at the same time that they adapt it to their particular needs: they appropriate it i.e., use it in unanticipated ways, and may also customize it i.e., tailor its functionality to make it work in a different way [33]. As customizations change the capabilities and limitations of an app, they influence user behavior over time. In this study, we analyze customization practices in ecosystems of communication apps through the lenses of co-adaptation to understand the value that people place on their customizations and how these influence their communication behavior.

We review the related work on customizations to mobile communication apps, highlighting that most work describes how users adapt to communication technologies, and that literature about

\footnotetext{
${ }^{1}$ Reactions are emojis that can be attached to a message after it was sent, as illustrated in [65]. Some of the apps that support reactions offer a fixed set of emoji options to react with (e.g. Messenger offers 7 emojis, including the heart eyes and thumbs up while others let users react with any pre-existing or custom emojis (e.g. Slack).
}

Proc. ACM Hum.-Comput. Interact., Vol. 3, No. CSCW, Article 26. Publication date: November 2019. 
how they adapt it mostly centers on appropriation rather than explicit customization. Moreover, we review related work on mobile communication via app ecosystems.

\subsection{Adapting to communication apps}

People incorporate diverse technologies into their everyday communication and learn to express themselves through the means provided by software applications, which they leverage for managing impressions and maintaining relationships. Walther's hyperpersonal model of interpersonal communication [60] proposes that, compared to face-to-face interactions, CMC grants greater control over the impressions people convey to others: people can perform selective self-presentations where they carefully edit and plan their messages before sending them, revealing select aspects of themselves while concealing facial expressions and physical appearances. Today, CMC supports a wide range of means of expression such as emojis, stickers, animated GIFs, video and audio messages, and photo decorations, which expand the range of opportunities to selectively convey impressions and express emotions. For example, people adopt emojis for augmenting messages with emotional or situated meaning, adjusting their tone and making them more engaging to the recipient [8], or even masking their true feelings [27]. Users often convey messages by simply sending an emoji or sticker [67] and may also use them for functional or strategic purposes, e.g., to "look like a nicer person" or start a conversation [29]. Moreover, people adopt GIFs to communicate emotions, cultural knowledge [38], and full-body physical expressions [56]. Since some customizations expand the available means of expression, e.g., adding custom emojis and stickers to an app, people may progressively adapt their self-presentation practices as they customize their communication apps.

$\mathrm{CMC}$ also enables new ways of maintaining relationships. Mobile messaging via SMS and apps, e.g., WhatsApp, opened the door to "any time, anywhere" text-based communication. This encourages people to send smaller, more mundane messages to each other throughout the day, blurring the beginning and end of conversations and creating a state of continuous connected presence [30] or $d$ welling [42]. Instant messengers with online status information also let people remain aware of their contacts' routines, helping navigate availability and enhance social connections [40]. In the particular case of close relationships, communication partners "adapt their use of media to support the greater range of expression important to their relationship, as well as use multiple means of communication to support their tie." [22]. For example, couples communicate via multiple communication apps to express their care for each other in diverse ways [9] and also switch channels to regulate conflicts [47]. To expand the range of communication media that support close relationships, researchers have explored various novel technologies that seek to increase the perceived intimacy of daily communication, for example, by sharing intimate data [3,19-21], or conveying the effort that a person invests in communicating with another [25,26]. By studying how people shape technology rather than how technology shapes relationship-maintenance practices, our work seeks to generate novel insights about how people leverage communication apps to mediate intimacy.

\subsection{Adapting communication apps}

Customization has been a widely studied topic spanning diverse domains, such as accessibility [46], software development [17], multi-player games [15, 50, 64], word processors [24, 34, 44], and task efficiency improvement $[5,16,53]$. On the other hand, most research on customizing communication apps and social media sites focus on privacy settings [51, 58, 61], rather than on the myriad of customization options for exploring more personal forms of expression.

Mackay identified triggers and barriers to customizing software in work environments. For example, people may customize software after noticing patterns of repetitive tasks, seeing an interesting customization by someone else, or wanting to retrofit changes from systems updates [32]. On the other hand, people may avoid customizing when it takes too much time or when a configuration

Proc. ACM Hum.-Comput. Interact., Vol. 3, No. CSCW, Article 26. Publication date: November 2019. 
is too hard to modify. In the scope of mediated communication, people may have other motives and barriers to customizing their apps. For example, Oulasvirta and Blom [43] argue that, among other reasons, people customize the aesthetics of their phones to express emotion and identity in relation to others, and Zhou et al. [67] describe how users customize their stickers for shaping their self-presentation on WeChat. Our study continues in this line of research, characterizing the value of a broader range of customization options in personal ecosystems of communication apps.

People appropriate features of communication apps with their own meanings and purposes, pointing to potential opportunities for customization. For example, users vary the normative forms of emoticons to express nuanced emotions [45]: while :) means "happy", typing :)))) expresses "very happy"; while T_T means "crying", typing $\mathbf{T} \_$_ $\mathbf{T}$ expresses "crying a lot". People reinterpret emojis based on how they look [55], neglecting their standard semantics: for some, the "grinning face with smiling eyes" emoji by Google @ conveys "blissfully happy", while Apple's implementation of the same emoji (i) means "ready to fight" [36]. Moreover, people in close relationships may develop shared meanings around an emoji when it evokes an inside joke, e.g., often sending the "fish" emoji OA because "Fish is our nickname of each other" [63]. Beyond how people appropriate the features of communication apps to fit their need for creative expression, we are interested in how they explicitly customize their communication apps to establish personal forms of expression.

\subsection{Communicating via ecosystems of communication apps}

Recent work advocates shifting the focus from studies of a single communication app or social media site to ecosystems of communication platforms, to obtain a more ecological understanding of how media shapes interpersonal communication. Zhao et al. [66] argue that "as users increasingly mix and remix use of different communication platforms, focusing on only one channel may conceal important insights". They show that people consciously regulate boundaries across the diverse social circles that "live" within each of their social media platforms to meet the nuanced social needs that a single platform could not support, e.g., posting updates to hundreds of acquaintances but also making sure that important content reaches close friends. Through the lens of Goffman's self-presentation theory [18], they suggest that people consider diverse audiences and functionality, or technical affordances, across their entire ecosystem of social media platforms when deciding where to share particular content. DeVito et al. [12] further demonstrate the value of an ecological approach, describing how LGBTQ+ users balance content, audience and functionality across multiple social media platforms to avoid stigmatization while still expressing their identities.

While social media platforms mediate communication between one person and a broad audience, messaging apps such as WhatsApp and Messenger focus on private, one-on-one or group conversations. The studies mentioned above provide insights as to why people use multiple social media platforms but are less clear as to why people adopt multiple messaging apps. Nouwens et al. [41] show how similar applications, in terms of functionality, become unique through use as people build communication places: idiosyncratic constructs that define membership rules, emotional connotations, and perceived purposes that influence how communication happens within each app of a user's personal ecosystem. To preserve their perceptions of how an app "feels", they separate contacts within different apps, e.g., restricting WhatsApp for family and close friends, while using Messenger for other acquaintances. As relationships evolve, contacts may move between apps, re-shaping the definitions of each communication place in their app ecosystem. While Nouwens et al. [41] describe how the contacts in an app affect communication via other apps, Sleeper et al. [48] show that people often share the content generated in one app to the contacts and audiences of other apps. Moreover, they describe how participants preserved boundaries across apps while using multi-channel strategies for generating content with composite functionality or for reaching 
multiple audiences. We adopt an ecological lens to investigate how the diverse customization options and functionality of different apps affect each other.

\section{METHOD}

We conducted an interview study to investigate how users customize their communication apps and inform the design of new ways of making mediated communication more personal. We are particularly interested in the role that customizations play in the way people relate to others, and how they manage the differences in the available customization options across their apps.

\subsection{Participants}

We recruited 15 participants ( 8 women, 6 men and 1 non-binary) between the ages of 22 and 32 (mean = 27) all with occupational backgrounds related to interface design (P2, P4, P8, P10) and software development (P1, P3, P5, P6, P7, P9, P11, P13, P15), except for a mental health supervisor (P12) and an accountant (P14). At the time of the study, participants lived in Argentina (5), Finland (2), France (1), Germany (3), Switzerland (1), Canada (1) and the USA (2), and originally came from China (2), Argentina (5), Canada (2), France, Sweden, Russia, Germany, Hungary and the USA. Reported operating systems include Android (11) and iOS (4). No compensation was offered for participating in the study.

We purposely looked for "extreme users" of communication apps in order to gain a richer view of the the motivations, practices and problems related to customizations in the domain of CMC. In this study, we consider "extreme users" to be people that use multiple apps for their daily communication and have customized at least one of them. Prior research $[6,13,57]$ indicate that, while data collected from extreme users may not be fully generalizable, it does provide rich insights that help framing new opportunities for design. In our case, we looked for experienced users that could provide insight into the extremes of the range of customization practices that may affect everyday communication and inspire designs for making mediated communication more personal.

\subsection{Procedure}

We recruited participants via one author's Facebook page, asking people "What kind of customizations do you do to your communication apps?" We defined a customization as "any change to an app that makes it work differently to how it worked when it was first installed". We invited 11 people to participate based on whether they commented on the Facebook post with customization examples beyond simple changes, e.g., creating and uploading custom emojis to Slack or changing Messenger's emoji shortcut in different conversations. We recruited four additional participants via word-of-mouth. All but one interview were conducted online, using Skype, Hangouts or WhatsApp according to participants' preferences. The remaining interview was conducted face-to-face.

Each interview lasted between 30 and 70 minutes. We asked participants about their latest and favorite customizations to their communication apps, how they use such customizations with their closest contacts, e.g., best friends, and how they communicate with those same contacts via other apps that may feature different customization options. We also explicitly asked about recent frustrations regarding the different customization options available across their apps.

\subsection{Data collection}

All interviews were audio recorded. Some participants sent us screenshots showing configuration settings or conversations that illustrated their customizations in context. We collected demographic data via an online questionnaire. 


\subsection{Analysis}

One author conducted a thematic analysis of the interview data, first with an inductive (data-driven) and later with a deductive (question-driven) approach, as described by Braun and Clarke [4]. We analyzed the data from a constructionist perspective with a bounded relativism ontology [39]. Thus, we do not claim any absolute truths about participants' behaviour, and the results of our analysis reflect a shared understanding of their customization practices that we constructed together during the interviews. We also acknowledge that our analysis is influenced by our own use of communication apps and our broader cultural context.

One author first compiled 356 quotes and notes from the interviews and annotated them with codes. This initial coding phase resulted in 67 codes. Some of the most common codes were "customization for enhancing expression", "customization used for few special contacts", "selfpresentation via a particular sticker/emoji", "negotiation over shared customization", "breakdown" and "workaround". Two codes, "breakdown" and "workaround", were not consistently related to customizations but offered novel insights into how users managed the diverse means of expression available across their app ecosystems. We agreed to broaden our initial scope and re-coded the quotes and notes annotated as "breakdown" or "workaround" to better understand users' struggles around their preferred forms of expression (Vitale et al. [59] report a similar analysis approach.) After iterative discussions within the research team, we group the final set of codes into ten categories, organized into three themes.

We compiled the list of all reported customizations, shown in Figure 4 (Appendix A). We avoid reporting counts for stories related to specific customizations, since our methods focus on characterizing customization practices and their role in mediated communication rather than obtaining statistics about patterns of behaviour.

The next section presents a taxonomy of customization options in communication apps which serves to contextualize and interpret our findings. We then describe the value of customizations in mobile communication under three themes: how participants leveraged their customizations to develop personal forms of expression across their apps, the breakdowns that prevented them from expressing themselves as they preferred, and the workarounds they established so they could express themselves as desired, despite these barriers.

\section{TAXONOMY OF CUSTOMIZATION OPTIONS IN MOBILE COMMUNICATION APPS}

We created a taxonomy of customization options in communication apps to capture the key factors that influence the social dynamics between users connected via the same app. This helps contextualize the study results and helps us analyze how different types of customizations support users' personal forms of expression. The taxonomy describes customization options according to: Visibility-whether they are visible to others; Ownership-whether the user alone or other contacts control them; and Scope-whether they are applicable at the app, conversation, or ecosystem level.

\subsection{Visibility}

The results of customizing a communication app may be visible to the user's contacts or not.

Private customization options are only visible to the user who made them. For example, the user's contacts will not be aware of changes the user makes to WhatsApp's notification sound.

Social customization options are visible to the user and their contacts, which lets contacts react to and act upon the user's customizations. For example, if a user adds a new sticker pack to Telegram and sends one in a conversation, the recipient will see it and be able to add it to their own sticker collection. 


\subsection{Ownership}

When two or more users communicate via the same app, each may retain individual ownership over specific customization settings, or share ownership with other users to control the settings of a customization option in a collaborative way.

INDIVIDUAL customization options offer exclusive control to the user who made the customization (the most common type of Ownership). For example, two Messenger users can customize their sticker collections by adding sticker packs they like, and each user will have independent control over their own collection.

SHARED customization options give shared control to two or more users. For example, any two Messenger users can change the emoji shortcut at the bottom of their conversation (by default, Facebook's blue thumbs up) for a different emoji. Both will then have access to the new emoji shortcut and may modify it at will. SHARED customization options always have SociaL visibility but not all SOCIAL customization options are SHARED-most customization options that are mutually visible rarely allow mutual control. Figure 4 in Appendix A shows that most of the currently available Social customization options feature INDIVIDUAL ownership.

\subsection{Scope}

The results of a customization may affect only particular conversations, the whole app, or any conversation in a user's ecosystem of communication apps.

App customization options are settings that affect the app itself and may globally affect all conversations with the contacts in that app. For example, changing the background image in Telegram will result in the same background for all conversations.

CONVERSATION customization options affect only the conversation with one or many contacts. For example, unlike in Telegram, WeChat users can set a different background image for each individual contact or group chat.

ECOSYSTEM customization options exceed the scope of any single app and have the potential to affect conversations from multiple apps. These are not customizations to communication apps per se, but enrich the means of expression that users can access in their conversations within apps. For example, installing the Tenor Keyboard on their phone lets users search for animated GIFs from within the soft keyboard ${ }^{2}$ and send them via any app.

\subsection{Differences in customization options across communication apps}

We compiled a list of 22 customization options across 9 apps and categorized them according to their Visibility, Ownership and Scope. The full list is available in Appendix A.

Some customization options are app-exclusive or only available in very few apps (Figure 1), such as changing the emoji shortcut (Messenger) or adding a custom emoji (Slack, HipChat, Flowdock, all apps for workplaces). Others are available in multiple apps but work in different ways. Figure 2 shows a subset of the reported customization options to illustrate how different apps assign them a different Visibility, Ownership or Scope. For example, while changing the conversation background affects all conversations in WhatsApp and Telegram (App scope), WeChat users can also choose different backgrounds for specific conversations (Conversation scope). Another example is changing a contact's nickname: while WeChat allows users to set custom nicknames for their contacts throughout the app (Private visibility, IndividUAL ownership, ApP scope), Messenger allows users and their contacts to change each other's nicknames in the context of each conversation they join (Social visibility, SHared ownership, Conversation scope).

\footnotetext{
${ }^{2}$ Soft keyboards (or software keyboards) are digital representations of a keyboard to enable on-screen text input without the need of a physical keyboard.
} 


\begin{tabular}{|c|c|c|c|c|c|c|c|c|c|}
\hline Customization option & Messenger & WhatsApp & Telegram & WeChat & Instagram & Snapchat & Slack & HipChat & Flowdock \\
\hline Add a custom emoji & & & & & & & $\mathrm{x}$ & $\mathrm{x}$ & $\mathrm{x}$ \\
\hline Add a custom sticker & & & & $\mathrm{x}$ & & & & & \\
\hline Add a sticker pack & $\mathrm{x}$ & $x$ & $\mathrm{x}$ & $x$ & & & & & \\
\hline Add a custom sticker pack & & & $\mathrm{x}$ & $\mathrm{x}$ & & & & & \\
\hline $\begin{array}{l}\text { Change conversation } \\
\text { background }\end{array}$ & & $\mathrm{x}$ & $\mathrm{x}$ & $\mathrm{x}$ & & & & & \\
\hline Change color of chat bubbles & $\mathrm{x}$ & & & & & & & & \\
\hline Change emoji shortcut & $\mathrm{x}$ & & & & & & & & \\
\hline Change group chat title & $\mathrm{x}$ & $\mathrm{x}$ & $\mathrm{x}$ & $x$ & $\mathrm{x}$ & $\mathrm{x}$ & & & \\
\hline Change group chat picture & & $x$ & $\mathrm{x}$ & $\mathrm{x}$ & & & & & \\
\hline Change contact nickname & $\mathrm{x}$ & & & $x$ & & $\mathrm{x}$ & & & \\
\hline Change own nickname & $\mathrm{x}$ & $\mathrm{x}$ & $\mathrm{x}$ & $\mathrm{x}$ & $\mathrm{x}$ & $\mathrm{x}$ & $\mathrm{x}$ & $x$ & $\mathrm{x}$ \\
\hline Change contact profile picture & & (discontinued) & & & & & & & \\
\hline Set favorite contacts & & $\mathrm{x}$ & $\mathrm{x}$ & $\mathrm{x}$ & & & & & \\
\hline Change notification sound & $x$ & $\mathrm{x}$ & $\mathrm{x}$ & $\mathrm{x}$ & $\mathrm{x}$ & & & & \\
\hline $\begin{array}{l}\text { Change color of chat bubbles } \\
\text { and background (color theme) }\end{array}$ & & & $\mathrm{x}$ & & & & $\mathrm{x}$ & $\mathrm{x}$ & \\
\hline Add chatbot & & & $\mathrm{x}$ & & & & $x$ & $x$ & $\mathrm{x}$ \\
\hline Add custom chatbot & & & $\mathrm{x}$ & & & & $\mathrm{x}$ & & \\
\hline Change Slackbot settings & & & & & & & $\mathrm{x}$ & & \\
\hline Change status message & & $\mathrm{x}$ & & & & & $\mathrm{x}$ & $x$ & \\
\hline Install plugin (e.g. polls, games) & $\mathrm{x}$ & & & $\mathrm{x}$ & & & $\mathrm{x}$ & $x$ & $\mathrm{x}$ \\
\hline
\end{tabular}

Fig. 1. Reported customizations options and which apps support them. We include only customization options with CONVERSATION and APP scope since ECOSYSTEM customizations are found on soft keyboards, not within apps.

\begin{tabular}{|c|c|c|c|c|c|c|c|c|}
\hline \multirow{2}{*}{ Customization option } & \multirow{2}{*}{ Reported apps } & \multicolumn{2}{|c|}{ Visibility } & \multicolumn{2}{|c|}{ Ownership } & \multicolumn{3}{|c|}{ Scope } \\
\hline & & Private & Social & Individual & Shared & App & Conversation & Ecosystem \\
\hline \multirow[t]{3}{*}{ Add a custom emoji } & Slack & & $\mathrm{x}$ & & $\mathrm{x}$ & $\mathrm{x}$ & & \\
\hline & Flowdock & & $x$ & & $x$ & $\mathrm{x}$ & & \\
\hline & HipChat & & $\mathrm{x}$ & & $\mathrm{x}$ & $\mathrm{x}$ & & \\
\hline \multirow[t]{2}{*}{ Add a sticker pack } & Messenger & & $\mathrm{x}$ & $\mathrm{x}$ & & $\mathrm{x}$ & & \\
\hline & Telegram & & $x$ & $x$ & & $\mathrm{x}$ & & \\
\hline \multirow[t]{3}{*}{ Change conversation background } & WeChat & $x$ & & $x$ & & $\mathrm{x}$ & $x$ & \\
\hline & Telegram & $\mathrm{x}$ & & $\mathrm{x}$ & & $\mathrm{x}$ & & \\
\hline & WhatsApp & $x$ & & $\mathrm{x}$ & & $\mathrm{x}$ & & \\
\hline Change color of chat bubbles & Messenger & & $\mathrm{x}$ & & $\mathrm{x}$ & & $\mathrm{x}$ & \\
\hline $\begin{array}{l}\text { Change color of chat bubbles and } \\
\text { background (color theme) }\end{array}$ & Telegram & $\mathrm{x}$ & & $\mathrm{x}$ & & $\mathrm{x}$ & & \\
\hline Change emoji shortcut & Messenger & & $x$ & & $\mathrm{x}$ & & $\mathrm{x}$ & \\
\hline \multirow{3}{*}{$\begin{array}{l}\text { Change contact } \\
\text { nickname }\end{array}$} & Messenger & & $\mathrm{x}$ & & $\mathrm{x}$ & & $\mathrm{x}$ & \\
\hline & Snapchat & $x$ & & $x$ & & $x$ & & \\
\hline & WeChat & $x$ & & $x$ & & $\mathrm{x}$ & & \\
\hline \multirow[t]{2}{*}{ Add chatbot } & Slack & & $\mathrm{x}$ & & $\mathrm{x}$ & $\mathrm{x}$ & $\mathrm{x}$ & \\
\hline & Telegram & & $x$ & $x$ & $\mathrm{x}$ & $\mathrm{x}$ & $\mathrm{x}$ & \\
\hline Change Slackbot settings & Slack & & $x$ & & $x$ & $x$ & & \\
\hline \multirow[t]{2}{*}{ Add alternative soft-keyboard } & Bitmoji Keyboard & & $x$ & $\mathrm{x}$ & & & & $\mathrm{x}$ \\
\hline & Tenor Keyboard & & $x$ & $x$ & & & & $x$ \\
\hline Add stickers to soft-keyboard & Gboard & & $\mathrm{x}$ & $\mathrm{x}$ & & & & $\mathrm{x}$ \\
\hline
\end{tabular}

Fig. 2. Subset of reported customization options and apps, categorized by Visibility, Ownership and Scope. Grey cells highlight differences across apps. (See Appendix A for the complete list.)

Next, we refer to our taxonomy of customization options to indicate how different levels of Visibility, Ownership or Scope support diverse personal expressions. Moreover, we discuss the implications of the varying availability and taxonomy levels of customization options across apps. 


\section{CUSTOMIZATION PRACTICES IN COMMUNICATION APPS}

Participants customized their communication apps to express aspects of their identities (e.g., interests, physical traits), intimate bonds within close relationships (e.g., inside jokes, shared activities), and organizational culture within work teams (e.g., norms around gender-neutral language). They expressed aspects of their identities primarily through INDIVIDUAL customization options with APP scope and Social visibility. SHARED customization options with CONVERSATION scope mostly supported expressions of intimate bonds within close relationships, and SHARED customization options with App scope supported the expression of organizational culture.

\subsection{Expressing identity}

SociAL customizations of INDIVIDUAL ownership enabled participants to express aspects of their identities within and outside conversations. For example, P15 liked sending stickers of "ALF" (Figure 3a) in response to messages on Telegram because his friends call him that and he identifies with the lovable, funny personality of the character. P6 preferred using stickers of the US-based singer Britney Spears rather than emoji on Telegram, reinforcing her image as a devoted fan. And P11 added a custom Nyan cat emoji (Figure 3b) to one of her Slack teams to decorate her nickname: "I wanted it next to my name and there was no Nyan cat emoji. I like cats and I like Nyan cats, and it's very me".

Customizations also let participants express different identities to different social circles within the same app, similar to how people manage impressions in face-to-face encounters [18] or how they select stickers and emojis based on the receiver [67]. For example, P9 installed a sticker pack in Messenger featuring a cartoon of a deer, but only used them with close friends; for the rest, he kept a more sober image by using the default "yellow face stickers". P11 decorated her nickname with a Nyan cat in one of her Slack teams, but chose a different emoji for her work team. Since changing her own nickname is an App customization and Slack treats teams as separate apps, she was able to present herself in different ways to different groups of people:

I have like three big balloons in my hand [in the profile picture for the Slack work team], so I have the balloon emoji ${ }^{\natural}$ ] next to my name. I thought of adding it [the Nyan cat] to my work team as well, but they like that I have the balloon emoji, because I'm somehow the balloon person on Slack. (P11)

\subsection{Expressing intimate bonds within close relationships}

Customizations helped participants express and reinforce intimacy within close relationships. Participants shared certain APP-scoped customizations with only the few contacts who found them meaningful. For example, P2 said that before graduating, she took many embarrassing pictures with her classmates, which they turned into custom stickers in WeChat and use only among themselves: "For one or two weeks, our group chat was full of everybody's ugly faces. I still have some of those emojis, but I'd use them specifically in the group of my bachelor's friends".

SHARED customizations with CONVERSATION scope played an important role in creating shared forms of expression with special contacts. This type of customization option invited participants to reflect upon what makes their relationships unique and customize their conversations accordingly. For example, $\mathrm{P} 4$ changed the Messenger emoji shortcut to a bike emoji $\sigma^{\circ}$ with one of her best friends. This became a special code between them: "We both bike and we talk about bikes a lot, so I put a bike there. When you don't have anything to say or it's an obvious answer, you just send a bike." P12 changed his Messenger emoji shortcut for a friend, facilitating a make-believe conversation style unique to them: "With my friend [name], the emoji for our conversation is this [the "pouting" emoji - ] and we send it as big as we can when you hold it down. It's more like we just pretend to be 

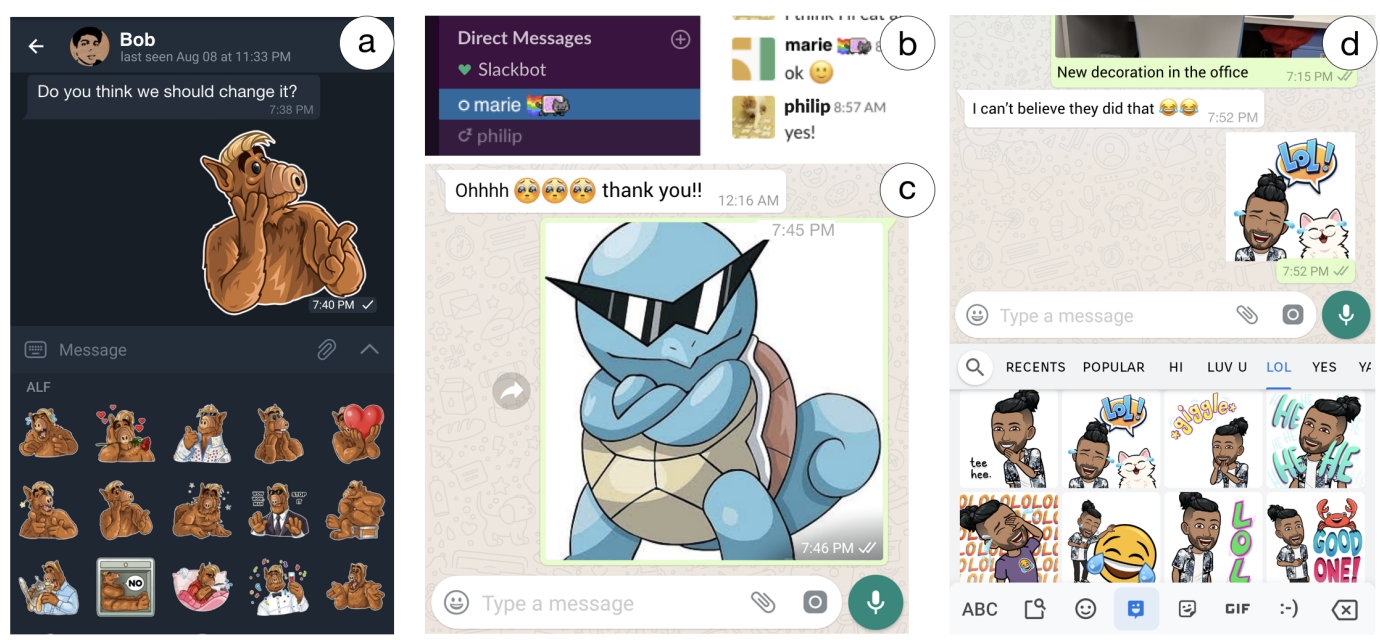

Fig. 3. Custom stickers or emojis that participants used to express their identities (images do not show real screenshots from participants to preserve their anonymity). (a) Stickers from the 1980s sitcom "ALF" on Telegram used by P15 because his friends call him "Alf" and he identifies with the character. (b) Custom Nyan cat emoji (a popular Internet meme) next to P11's name in one of her Slack teams. (c) Pokémon "Squirtle" wearing sunglasses (another popular Internet meme) is P5's signature sticker and custom emoji in all of her apps, sent as an image on WhatsApp. (d) Bitmoji stickers in the GBoard keyboard, personalized with an avatar that resembles P13's physical appearance, used across all his apps.

angry about things and our conversation revolves around that a lot”. P3 described how, together with a close friend, they customize each other's nicknames on Messenger to reflect their inside jokes:

We change each other's Facebook [Messenger] names often. So I'm Costco artisanal bread because one day I was buying this bread and it was really good, so I sent him a picture "Look at this bread! It's... artisanal! But it's from Costco...! And it's so good". I changed his to Innovator because he always like... makes this very stupid jokes, like "I'm so innovative! Disruption!" and stuff. So I think nicknames above anything is a sign of higher intimacy, because it deals directly with the person's identity. (P3)

Customizing an individual Conversation also reflected and increased feelings of connectedness, distinguishing one special contact from the rest: "I like changing the chat color and the default emoji, it's like a sign of closeness and intimacy. Like, here are my actual friends, people I talk to on a regular basis. It creates a differentiation from the default blue, the default [Facebook] Like thing" (P9). Customizations may increase feelings of connectedness especially when they represent the participant's intimate knowledge of each other or the nature of the relationship:

I changed the conversation to green [in Messenger] with my husband because he likes green. I also changed the thumbs up [emoji shortcut] for a kiss. I open the conversation with him, I see the emoji with a kiss and... every time I see it, it makes me feel good, makes me feel connected to him. (P7)

\subsection{Expressing organizational culture}

Customizations helped work teams develop a communication culture. Slack treats customization options such as adding a custom emoji or a chatbot as SHARED customizations with App scope, which means that all contacts within a Slack team can use and change them. 
For example, custom emojis added by one person can later be adopted by others, so everyone can contribute to the team's communication culture:

One of the GIFs I made was a sheep. Its head would disappear, and then it'd show the head of a bear. This comes from a popular phrase in Finnish culture, "a wolf in sheep's clothes", and the bear is like the mascot of the local cheap beer, which is the beer that we usually had at the office. It was surprising to see that other employees loved it so much that they used it to react to negative news, surprises, and a lot of things. It was very good for the social culture inside the office. (P1)

Customizing the Slackbot-Slack's default chatbot that reacts to a list of customizable words and phrases-also offered opportunities for influencing the culture within the team. For example, P11 and some colleagues customized the Slackbot for encouraging a more gender-inclusive language in the office, which triggered a debate and brought awareness to the issue:

There was this conversation about... should you call people guys? Or is that noninclusive? Should we add this bot to say "guys is not gender neutral" or "you should just say people"? And then people who hadn't been at that conversation got a bit strange when it got triggered to them. So that started a conversation: should we have this for everybody or not, because not everyone feels excluded if you say guys. But others were like "oh! I never thought about that, thanks so much for pointing it out". (P11)

\subsection{Maintaining consistent expression across communication apps}

Some participants felt particularly attached to customizations that expressed their identity, motivating them to adapt each of their apps to support them by reproducing App-scoped customizations. For example, the "Squirtle with sunglasses" (Figure 3c) was P5's signature emoji/sticker, which she used in all her apps instead of the regular sunglasses emoji: she added a sticker pack of Pokémon in Telegram and added a custom emoji in Slack to use it in reactions, and also kept an image on her phone's media to send it via WhatsApp. Customizations scoped to the entire Ecosystem of apps helped maintain cross-app identity expressions without having to adapt each app separately. For example, P13 added Bitmoji stickers (Figure 3d) to GBoard (Google's soft keyboard) to customize his stickers once and send them via any app.

Participants also maintained consistent expressions of intimate bonds with others by reproducing customizations with Conversation scope. For example, P11 had a group chat on Messenger and on Instagram integrating the same people, and she customized the title of both groups to be the same. P13, who talked with his friends from work via WhatsApp, Telegram and Slack depending on the purpose of the communication, imported some custom stickers with pictures of themselves from Telegram to WhatsApp as soon as WhatsApp added support for stickers (November 2018).

In summary, we found that participants customized their communication apps' to express aspects of their identities, intimate bonds within close relationships, and organizational culture. Beyond this, they also reproduced customizations across multiple apps to maintain consistent expressions of their identities and intimate bonds with others across their entire app ecosystems.

\section{EXPRESSION BREAKDOWNS IN ECOSYSTEMS OF COMMUNICATION APPS}

Many participants were frustrated by their inability to preserve their carefully crafted forms of expression across their ecosystems of apps. As they all used multiple apps to communicate, they often missed the customizations they did to an app when using another. We call such episodes expression breakdowns: frustrations around barriers to users' personal forms of expression, which often manifest as unmet expectations about the means of expression that an app should support. Expression breakdowns shift a person's focus of attention from their communication to the app

Proc. ACM Hum.-Comput. Interact., Vol. 3, No. CSCW, Article 26. Publication date: November 2019. 
limitations that prevent them from expressing themselves as they want-similar to how technical breakdowns shift the focus from a task to the workings of the technology [62]. The main sources of expression breakdowns centered around app-exclusive customization options and different levels of Visibility, Ownership and Scope between apps, as well as other non-customizable means of expression such as app-exclusive stickers.

\subsection{App-exclusive customization options}

Breakdowns around identity expression related primarily to apps lacking (custom) stickers ${ }^{3}$. For example, P6 missed her Britney Spears stickers from Telegram when talking to her friends in WhatsApp, similar to P15 and his "ALF" stickers.

The uneven support for stickers across apps also prevented expressions of intimate bonds and shared interests with others:

I have a lot of doggy emojis [stickers] in WeChat. And I'm so used to them. Sometimes when I chat on WhatsApp or Messenger and I talk with friends, and I know they also like dogs, I'm like "Oh, I wish I could just... like, switch apps" (P2).

Customized contact nicknames helps distinguish close relationships from other contacts, but only some apps support this. P12 added prefixes to his contacts' nicknames in Snapchat to create ad-hoc categories of people. He explained that he has many contacts in both Snapchat and Instagram and prefers sending private messages to broadcasting content to everybody. Adding prefixes to people's names helps him find his closest contacts faster. Instagram does not support changing contacts' nicknames, which discourages him from sending private messages on Instagram-a more intimate way of communicating than broadcasting content:

I use Snapchat to send [pictures] to a ton of different people so like I actually put letters in front of everybody's name so that they're organized by group, and then if I'm gonna send it to family I just go to F and then click everybody. Or like triple A is the people I send things to all the time, one singular A is like my best best friends. (...) I can't make those categories that I make in Snapchat on Instagram and it's extremely frustrating, it would make life a lot easier. That's why I don't send things [via private messages] commonly through Instagram, because I haven't been able to organize it that way.

Some participants complained about app-exclusive customizations that enhance usability rather than expression, such as changing the color theme of an app to a dark mode: "Telegram has it [night mode theme] and for me it's vital, I have sight issues. I figured, if Telegram has it, WhatsApp will have $i t$ " (P5). We believe that such customizations may still influence users' preferences of where to initiate conversations and degrade the experience when communicating via other apps

\subsection{Customization options with design differences across apps}

Differences in the design of a customization option can discourage participants from reproducing customizations across apps, especially when they prevent cross-app expressions of bonds within close relationships. For example, P9 changed the color of the chat bubbles in conversations with close friends in Messenger because it felt more personal that way: "It has a personal touch, like when you change the tone of your voice or change your mood. I think it's a way of translating that to text communication. That intimate feeling... as if you were interacting physically with a person". However, he felt it was not worth it to change conversation backgrounds in WhatsApp or Telegram: "I was quite disappointed, I thought it was actually conversation based, so it's not very interesting".

${ }^{3}$ WhatsApp had no support for stickers until late 2018. 
Moreover, differences in the Scope of notification settings can interfere with prioritizing messages from specific contacts such as friends and romantic partners:

In WhatsApp you can change notification settings, I wish I had that on Messenger. When I was an intern at [company] we had a personal group in WhatsApp. I set the notification light to blue, then I knew it was somebody on that group that was trying to reach me. Or if it's someone you're dating, you want to know how fast it needs replying. How much of my attention I should bring to it. (P11)

In such cases, participants felt that customization options with CONVERSATION rather than App scope are worth customizing for expressing different bonds with different people. Others referred to the Ownership of the customization option rather than its Scope, for example, preferring customization options with SHARED rather than INDIVIDUAL ownership for expressing connection with someone else:

I know that in WeChat you can change the chatting background with someone specifically, I think it's even more like... creating a room. But I didn't do it. I guess I don't feel the need. It's something difficult to share. I noticed that on Messenger one friend changed the bubbles to green, so... that was a surprise and it's also actually nice because when I'm chatting with him it's different, like a new app. But that was his change, and it also affected my interface. It feels like talking to him it's something very special, like a customized chatting room with him. (P2)

Note that these preferences vary greatly across participants. For example, P5 was glad that Telegram's background images were PRIVATE customization options of INDIVIDUAL ownership, because her boyfriend used a picture of their dog as their conversation background but she preferred the default: "It drives me crazy... having a picture with my texts feels like a 90s thing".

Other expression breakdowns involve stickers that are "trapped" in one app and cannot be used in others, even if those apps support stickers. Most such apps let users install sticker packs only from their own sticker stores, leading to expression breakdowns related app-exclusive sticker packs. For example, some participants restricted their use of stickers to the apps that offer those they identify with: "I use stickers on Telegram but not on Messenger. I don't know who creates the stickers for Messenger but I don't like how they look. On Messenger they're like smileys from cartoons. On Telegram it's different, for example Angela Merkel or Putin" (P7).

Finally, some app's usability problems discourage participants from reproducing customizations across apps. In the worst cases, participants avoided usability issues and were thus unable to express their identities and intimate relationships as they would have liked. For example, when WhatsApp added support for stickers, P13 tried transferring stickers of himself and his colleagues from Telegram; however, this required too much effort so he only migrated a few: "The best you can do on WhatsApp for sharing stickers is sending one by one, and the other person has to add one by one. We migrated maybe 3, 4 stickers but it's a pain, so we stopped”. P4 used the Bitmoji soft keyboard (which provided cross-app access to her Bitmojis) only with special contacts, since she felt it supported "a different level of connection". However, she stopped using it on WhatsApp:

It became really annoying because every time you send a sticker or someone sends you a sticker it gets saved on the phone, on the conversation's media. I think that's one of the main reasons I didn't use it anymore. (P4)

\subsection{App-exclusive means of expression}

Beyond customizations, participants also experience expression breakdowns around means of expression that, despite not being customizable, help communicating in more personal ways, e.g., emojis, reactions, and audio messages. Some developed habits of expression around app-exclusive 
features and felt frustrated when they had to adapt their expression to the limitations of each app. For example, many missed Slack's reactions in other apps: "Reactions are very powerful because you don't break the communication. I'd like reactions on Telegram, and WhatsApp too" (P6). While Messenger and iMessage also support reactions, Slack allows users to react to messages with their own custom emojis. P1 used to create custom emojis for Slack but stopped doing so when he changed jobs and switched to Flowdock (another app for teams), only because he could not use them as reactions: "Flowdock allows you to add your own emoji, but it doesn't really encourage me to do it. It doesn't have reactions like Slack, so emojis only appear in the line of your message".

One participant adopted a strategy in which he restricted his forms of expression to the most common features across apps. However, he still struggled with unmet expectations about what every app in his ecosystem should support:

I feel that when I want to send a thing, I think in terms of an image, a GIF, an audio... which fits in any of the applications. I usually mold my mind to express myself in one of those things I know I can do. And I find particularly bothersome when I want to send an audio via Hangouts [which lacks support for audio messages]. I hate it when somebody from work talks to me via Hangouts and I'm walking on the street. I want to reply with an audio. (P15)

Many participants miss particular emojis during a conversation, even though all apps support emojis. This occurs when two apps visually represent emojis in different ways i.e., the same Unicode character shows different pictograms. Although all emoji implementations try to preserve the same semantics, users may assign their own meaning to an emoji based on how it looks [27], and sometimes re-purpose them to convey secret codes with close friends or romantic partners [63]. Thus, different emoji representations may prevent consistent forms of expression within close relationships across apps. For example, P12, whose conversations with a close friend revolved a lot around the "pouting" emoji 2 on Messenger, explained that he gave up the joke on Instagram where the emoji looks different: "I don't use it much on Instagram because it's that faded yellow to red [.] instead of the foam red face. I want it to be red" (P12). P14 had become very attached to the "raising hands" emoji on WhatsApp after a painful breakup, but could not find it on Instagram. She explained that, to her, the yellow hands and the "tiny sparks" signified hope and encouragement and they had become one of her most used emojis, especially in conversations with a friend that helped her overcome the breakup:

When I chat on Instagram I can't use emojis that I'm deeply attached to. For example, the "little hands" [i.e., raising hands emoji it looks different [a person raising their arms $]$ ].

Participants also complained about differences not only between their own apps but also between their apps and their closest communication partners. Some apps use the operating system's emoji implementation rather than their own, so an iPhone user chatting with an Android user may see different representations for the emojis they send to each other [37]. P1 explained that after an update, his emojis on WhatsApp changed but his girlfriend's did not. He felt the new emojis expressed different emotions than the earlier ones and described how he wanted to customize his set of emojis to fix the problem:

I would change the emoji back to the old ones if I could, but I wouldn't use any custom ones that only I can see, since then I wouldn't be sharing the experience with her - it would be different. So now I still send her some of the affection related emojis like in the old days sometimes, but it's mainly for her benefit. (P1) 
In summary, participants experience diverse expression breakdowns when trying to express their identities and intimate bonds with others in consistent ways across apps. The key reasons include: app-exclusive customization options, customization options with design differences across apps, and app-exclusive means of expression.

\section{WORKAROUNDS TO EXPRESSION BREAKDOWNS}

To overcome expression breakdowns, participants sought workarounds that let them maintain some of their personal forms of expression consistently across apps. Some workarounds involve finding alternative means of expression within the current app, whereas others rely on the means of expression and customizations of other apps. Participants' arduous, inventive workarounds illustrate how important it was for them to preserve personal forms of expression across their entire ecosystems of communication apps.

\subsection{Selecting alternative means of expression within the current app}

Some participants opted for finding a compromise between the ideal forms of expression for a conversation and what they could convey with the available features in the current app. A recurrent workaround for Telegram users who enjoyed sending stickers was to send similar GIFs on WhatsApp For example, P6 described how she regrets being unable to use her Britney Spears stickers on WhatsApp: "I tell my friends "C'mon! Let's move to Telegram [i.e., abandon WhatsApp for good] so I can send you stickers... really!" So I send them GIFs". However, some felt that searching for GIFs was less practical than accessing their sticker collection. P2 explained that when she lacks access to her shiba dog stickers she considers searching for equivalent GIFs. However, this requires additional effort because apps only support search functions for GIFs and users cannot save them for future use, which discouraged P2 from sending GIFs to substitute her shiba dog stickers. P6 experienced the same problem but found a way of collecting her favorite GIFs: "I have a repository of GIFs [in my phone's media library] that I always use because I don't want to be searching". These examples further illustrate participants' idiosyncratic expression styles: their goal was not simply to decorate their messages with any stickers or GIFs, but to consistently use those that best represented them.

Other workarounds attempted to reproduce the representation of an intimate bond with a specific contact from one app in another:

I added an air horn emoji [to Slack]. This is because [Bob, a colleague] and I are very good friends too, and talk half on Messenger, half on Slack. Sometimes in conversations with [Bob] I air-horn-react to the stupid memes he sends on Slack, but I also remember sending him an image of an air horn or an MP3 of the air horn sound on Messenger. (P3)

\subsection{Moving conversations to another app}

Participants sometimes failed to find alternate forms of expression within their current app and decided to move the conversation to an app that better suited their needs:

I have one of my closest friend who I talk with all the time, and we both use the little hands ["raising hands" emoji continue on [move to] WhatsApp, just for the silly reason of [using] the little hands.

Unfortunately, this workaround means the loss of the initial conversation's context, which discouraged many participants. For example, P5 was fond of a Telegram bot that creates polls in a group conversation and easily collects votes. However, she avoided switching apps when she had to negotiate decisions in other apps without the feature:

A couple of times we had to decide something on Slack but didn't have polls. But we prefer to stay in Slack and just list the options and say "I like this, I like that". It's a 
bit chaotic, but I don't want to go to another application outside of where I am. The context switch is a waste of time, and when we have a situation where we have to vote, it's something we want to settle quick. (P5)

\subsection{Obtaining expressive media and generating content outside the current app}

A few participants invented clever, if arduous, ways to leverage exclusive features from one app to use them in another. They considered the customizations and means of expression available across their entire communication apps ecosystem when composing messages.

Some participants took a screenshot of a sticker in one app, cropped it to fit the sticker, and then sent it via another app. For example, P9 took a screenshot of one of his deer stickers in Messenger for sending it as an image on WhatsApp, although only "once or twice because it takes too much work". P15 found a more efficient approach and installed a Telegram chatbot to convert his stickers to images to be able to send them via WhatsApp:

If I'm in the need, I use a Telegram bot called "sticker downloader" which converts stickers to images and lets me send the sticker via WhatsApp. For example, one time the sticker I wanted to share was a sticker that someone had put in a sticker collection specific to my University, which had inside jokes fun to share. (P15)

Beyond stickers, some participants went out of their way to switch apps to access a particular function, generate message content, save it as a file, then send it via the current conversation app:

Snapchat seems to integrate them [Bitmoji stickers] really well. So because the other apps don't really let me put a Bitmoji on a picture and I want that, if I'm sending it to somebody through Instagram who doesn't use Snapchat, then I'll use Snapchat, put the Bitmoji on the picture, save that, then upload it to the other app. (P12)

Such efforts were sometimes dedicated to a special person. P12 had recently applied a similar workaround to send a Bitmoji-decorated picture to a close friend on WhatsApp. His friend no longer used Snapchat but P12 still had access to "her dead account" which he used for generating decorated pictures with Friendmojis-Bitmoji stickers of the user and their friend's avatars together. Note that WhatsApp and Instagram offer functionality for decorating pictures with stickers, but only Snapchat supports picture decorations with the user's customized Bitmojis.

Participants also accessed features from other apps to suit individual expression needs. For example, P13 and his colleagues had created a custom chatbot on Slack to generate an image of a meme with a custom text: when sending a phrase to the bot, it would reply with a meme featuring that phrase with all vowels replaced by the letter " $i$ ". They used it for when people complained about unimportant things. During a conversation on WhatsApp with a friend from outside work, P13 saw a great opportunity to use that meme: "This guy complained because he cannot see WhatsApp stickers on his smartwatch. I mean, that was THE use case for the [meme chatbot], so I took the trouble of going to Slack, [generate a meme with with the chatbot based on my friend's message], send the image to myself, and then send it on WhatsApp”. P12 applied a similar workaround, not for compensating missing functionality in one app, but to access his preferred "flavour" of a feature:

"Rewind" [a video effect for making videos that play backwards] works better on Instagram, so sometimes I'll do that [post a Rewind video on Instagram], but usually save it and put it on Snapchat. (P12)

\section{DISCUSSION}

We conducted an interview study to inform the design of more personalized mediated communication, guided by the following research questions: 1) What customizations do people make to their communication apps, and how can we characterize them so as to inform design? 2) What value do 
people place on the diverse customization options available throughout their personal ecosystems of communication apps? To obtain rich insights into diverse customizations, we interviewed 15 "extreme users" that used multiple apps for their everyday communication and customized at least one of them.

We identified 22 customization options from nine different apps that participants leveraged for expressions of identity, organizational culture and intimate bonds within close relationships. We characterized their customization practices by creating a taxonomy of customization options in communication apps. Next, we discuss the implications of diverse intersections of our taxonomy for $\mathrm{CMC}$ for maintaining relationships online. We also discuss how the customizations to one app may influence how users express themselves via other apps, contributing to our understanding of ecosystems of communication apps.

\subsection{Customizing as a relationship maintenance practice}

Our findings offer rich evidence of how customizations can play an important role in maintaining relationships, mainly by granting more control over self-presentation strategies and offering opportunities for expressing and reinforcing intimacy within close relationships. As reviewed in Section 2.3, previous studies on ecosystems of communication platforms report that balancing content and audience are key parameters in people's self-presentation strategies: they inform what to share on each platform in order to manage different impressions within different social circles [12, $41,48,54,66]$. Here, in addition to content and audience, participants leverage customizations to embed aspects of their identities and relationships into the apps themselves, turning them into more intimate places for communication and enabling more personal self-presentation strategies.

We introduce a taxonomy of customization options to capture design axes that may affect interpersonal communication: Visibility determines whether a customization option is private or visible to the user's contacts; Scope defines whether it affects a single conversation, an entire app or communication across multiple apps; and Ownership defines whether control over settings is restricted to the user or shared with their contacts. Each axis suggests implications for maintaining online relationship

The Visibility of a customization option determines whether it plays an active role in interpersonal communication. Customization options with PrIVATE visibility allow participants to discreetly organize and prioritize their contacts (e.g., P12 renaming contacts on Snapchat for categorizing them into separate audiences), as well as tweak their apps for practical purposes not directly linked to communication, e.g., P6 setting a dark theme on Telegram to take care of her eyes. By contrast, participants use customization options with Social visibility to express their identities and manage impressions, e.g., P15 sending "ALF" stickers as a reference to his name, but also to convey a funny, lovable personality.

The Scope of a customization option (CONVERsation, App, Ecosystem) influences which audience the user considers when customizing, especially when featuring Social visibility. Customization options scoped to a Conversation usually evoke aspects of a particular relationship, e.g., P7 changing the color of the chat bubbles to his husband's favorite color, while those scoped to an entire App relate to expressions of identity that the user wanted to convey to all contacts hosted in that app, e.g., P11 adding the balloon emoji to her nickname on Slack. Customizations to soft keyboards let users affect any app in their ЕсозчsтEM, and mostly support expressions of identity that the user considers relevant to different audiences across apps, as well as towards contacts that span many apps, e.g., P13 using Bitmoji on GBoard to send stickers featuring an avatar of himself across all of his apps. 
The Ownership of a customization option (INDIVIDUAL, SHARED) determines its ability to express the identity of an individual or the shared bonds that identify a dyad or group. INDIVIDUAL customization options support nuanced control over the impressions participants want to convey to others, while SHARED ones invite all communication partners in a conversation to collaboratively express intimate bonds that flag their relationship, rendering the customization an intimate communication act in itself.

Some of the most interesting insights appear at the intersections of these axes. Our data suggest that different combinations of Visibility, Scope and Ownership support diverse relationship maintenance practices. For example, customization options with SHARED ownership and CoNVERSATION scope, which are always Social, invited participants to dedicate customizations to particular people. They selected these customization options only for their closest contacts, which some treated as a indication of greater intimacy, e.g., P9 changing Messenger's chat-bubbles color only for his actual friends. When ownership was SHARED, participants collaborated with their closest contacts to add meaning to their customizations, decorating their conversation space to reflect the elements that made their relationship special. Wiseman and Gould [63] describes how people in close relationships often change the meaning of particular emojis to express their "micro cultures", e.g., shared understandings, inside jokes. Here, participants materialized their "micro cultures" into customizations they co-owned with their closest contacts. Embedding inside jokes into nicknames (P3) or turning the shared love for biking in an emoji shortcut (P4) resulted in persistent symbols that reflected and reinforced the intimacy of the relationship. These insights invite us to revisit the notions of "connected" presence [30] and dwelling in messaging apps [42]: people in close relationships not only reaffirm their connection by exchanging small but frequent messages throughout the day, but also by customizing and embedding reminders of their intimate bonds into the apps that mediate their everyday communication.

When the scope of a SHARED customization option is applied to the whole App and not a single CONVERSATION, as with Slack, customizations help represent and build group culture rather than express bonds within close relationships. Creating custom emojis based on the workplace culture, e.g., P1's emojis featuring the company's mascot, expanded the emoji vocabulary available to all team members, reinforcing a sense of group identity every time somebody used it in a message or as a reaction. The slackbot also invited team members to collaboratively materialize inside jokes or social norms into automated word-triggered messages, e.g., P11's "should you call people guys?" customization. Nouwens et al. [41] propose that a user's experiences with the contacts in an app shape how the user perceives its "placeness", which in turn influences their future actions and experiences in that app. Here, we show how participants and their colleagues made this "placeness" explicit in the tools that mediate their everyday communication, promoting a communication style that fits their group culture.

The intersection of SocIAL customization options with App scope and INDIVIDUAL ownership allows for nuanced self-presentation strategies. Participants leveraged customizations to profile elements that were always visible, e.g., P5's pokémon emoji next to her Slack nickname, to convey a particular impression to the audience (all contacts) in a particular app, similar to how people tailor social media content to the perceived audiences in different platforms [12, 35, 66]. On the other hand, a stickers only becomes visible when the user decides to send it, serving as rich material for selective self-presention to others during a conversation [60], by carefully choosing which sticker to send, to whom and when.

Finally, participants leveraged Ecosystem-scoped customization options to selectively selfpresent to contacts from multiple apps, e.g., P13 using Bitmoji stickers via the GBoard keyboard across his communication apps and e-mail. While people often separate contacts in different apps and preserve distinct communication styles [41], our data suggests that they may also want to

Proc. ACM Hum.-Comput. Interact., Vol. 3, No. CSCW, Article 26. Publication date: November 2019. 
keep consistent forms of identity expressions across apps. We believe that Ecosystem-scoped customizations of this kind deserve in-depth studies in the future, as they promote expressions of identity that go beyond those possible in each individual app. Moreover, we believe that people may also value cross-app access to their personal forms of expression for their self-presentation in social media, where existing research focuses primarily on how self-presentation varies across platforms [54] rather than on users' efforts to express themselves consistently.

\subsection{Transferring habits of expression between communication apps}

An unexpected but important finding is the users' diverse efforts in maintaining consistent forms of expression across multiple apps. We call this phenomenon transfer of habits of expression within ecosystems of communication apps. We next explore this phenomenon through the lens of coadaptation [33] and discuss our contributions to theories of CMC and software customization.

Combining insights from the literature and our study suggest that people co-adapt their expression according to the capabilities and limitations of each app that mediates their communication. When they co-adapt to a particular app, they learn to express themselves in terms its functionality, e.g., reacting to a message with a GIF [23], and they appropriate these features with their own meanings and purposes, e.g., repurposing emojis within close relationships [63]. Our data show that people with strong needs for expression may also explicitly customize a communication app to enable more nuanced, personal ways of maintaining relationships online. As they learn to use, appropriate and customize a communication app, they nurture habits of expression based on both the available functionality and their own modifications to it, e.g., P5 expressing emotions with Britney Spears stickers, not just any sticker.

When looking at how participants co-adapt multiple communication apps in parallel, we see that the habits of expression nurtured in one app transfer to others. We see evidence of this in three ways:

First, as described in Section 5.4, some participants customized an app to enable forms of expression they acquired from other apps, e.g., P5's different versions of the Squirtle pokémon across apps. Others made Ecosystem-scoped customizations to soft keyboards to let them access personal sticker collections from within any of their apps.

Second, when customization was impossible, participants experienced expression breakdowns: frustrations related to their inability to express themselves in ways they learned from another app. Participants complained about app-exclusive customizations and other means of expression, such as particular sticker packs that they missed when communicating via other apps, even when those apps were for different contacts and purposes.

Third, some participants discovered inventive, effortful workarounds to recreate means of expression from one app into a conversation, by using one app to generate and export content and sending it via another app so as to express themselves in their preferred way, e.g., P9 taking a screenshot of a sticker on Messenger to send it via WhatsApp, P12 decorating a picture with Bitmoji on Snapchat to send it via Instagram. Other workarounds involved moving the conversation to the app with the preferred means of expression, or settling for the available means of expression that evoked a preferred one from another app.

These insights contribute to the ongoing discussion of how communication apps and social media platforms in an ecosystem affect each other [10-12, 41, 48, 66]. As mentioned in Section 2.3, recent research argues in favor of studying personal ecosystems of communication platforms rather than focusing on particular ones, showing how the contacts in one app may influence communication patterns in other apps [41]; how content shared with audiences in one app often comes from other apps [48]; and how people consider the entire set of affordances in their ecosystems of social media platforms when deciding what to share with whom [66]. Our work contributes new evidence about 
how individual apps shape communication via other apps: the habits of expression nurtured in one may transfer to others, thus affecting how people learn, appropriate and customize the diverse communication apps in their ecosystems. As people adopt more communication apps, they may develop new habits of expression that re-shape the way they express themselves in their other apps. This implies that the self-presentation and relationship maintenance practices a person performs in one app may not only be influenced by the contacts and functionality within that app, but by how they express their identities and intimate bonds with others in other apps as well.

Our findings also suggest new triggers and drivers for software customization in the context of CMC. When customizing their communication apps, participants were not trying to perform tasks more efficiently or avoid repetitive actions [32]. Instead, they customized to enable more personal forms of expression which they leveraged for self-presentation and relationship maintenance purposes. Moreover, participants' expectations and efforts related to transferring habits of expression between apps suggest that the way they co-adapted one app shaped how they co-adapted others, i.e., how they learned to use them, e.g., P15 assuming that if one app supports audio messages, others would as well; how they appropriated them for idiosyncratic uses, e.g., P12 using Snapchat as a content-generating app rather than a communication channel; and how they customized them, e.g., P13 installing stickers from Telegram in WhatsApp.

Our work also contributes to our understanding of how people perceive and manage the sociotechnical gap in their ecosystems of communication apps. Ackerman [1] presents the socio-technical gap as a central challenge for the CSCW community, defining it as "what we know we must support socially and what we can support technically". He argues that human activity is fluid, nuanced and contextualized, but that technology lacks the flexibility required by social life. Zhao et al. [66] proposed that people adopt multiple social media platforms in an attempt to close the sociotechnical gap in their ecosystems of social media platforms, enabling more nuanced strategies for maintaining the boundaries between different audiences and having more control over their impression management. Here, participants' expression breakdowns suggest a gap between how they wanted to express themselves and how each app allowed and restricted their expression, which they tried to bridge by appropriating and customizing their multiple apps. This gap might be harder to bridge for less savvy users, who may overlook opportunities for adapting their apps to their preferred forms of expression as well as multi-channel strategies [48] to leverage features of one app for their conversations via others. We hope our work inspires future efforts in helping users develop more personal forms of expression across their communication apps and reduce barriers to transferring habits of expression.

\section{IMPLICATIONS FOR DESIGN}

We invite app designers and researchers to use our taxonomy of customization options in communication apps as a design space for exploring new ways of making mobile communication more personal. Next, we illustrate three approaches to exploring new opportunities for design.

\subsection{Alternating levels of Visibility, Ownership and Scope}

Changing the Visibility, Ownership and Scope of an existing customization option may lead to novel alternatives. For example, designers that want to encourage expressions of intimacy within close relationships could change the Scope of Slack's custom emojis (which feature SHARED ownership) from App to Conversation, which would allow two colleagues to develop their own emoji-based secret codes without others knowing.

Similarly, designers that want to support (selective) self-presentation practices could change the Scope of Messenger's emoji shortcut from Conversation to ApP and its Ownership from SHARED to INDIVIDUAL, offering a user a shortcut to an emoji they feel expresses their identity (e.g., P14's

Proc. ACM Hum.-Comput. Interact., Vol. 3, No. CSCW, Article 26. Publication date: November 2019. 
raising hands emoji $(1)$ across all the conversations via that app. Moreover, changing its Scope to Ecosystem (e.g., enabling emoji shortcuts in a soft keyboard such as GBoard) would allow users to access the shortcut across apps.

\subsection{Exploring empty intersections of Visibility, Ownership and Scope}

The current design space of customization options also reveals combinations of Visibility, Ownership and Scope that, to the best of our knowledge, are still unexplored (Fig. 4), such as SociaL visibility with SHARED ownership and ECOSYSTEM scope. We believe this combination presents an interesting opportunity for supporting close relationships and could help transferring their shared habits of expression between apps (e.g., two friends could change each other's nicknames for their conversations in all their apps). Such cross-app, SHARED customization options would require a unique representation of each contact across the app ecosystem. For example, instead of customizing the notification settings for "the Alice in WhatsApp" or "the Alice in Telegram", a user could simply customize the notifications for "Alice" valid for all her messages, regardless of the mediating app.

\subsection{Shifting from app-enclosed to user-owned means of expression}

We believe that beyond customizations, there is a need for cross-app access to users' favorite means of expression: we propose shifting from designing app-enclosed to user-owned means of expression that people can bring into each of their apps to maintain personal forms of expression. Similarly to how soft-keyboards such as GBoard support customizable collections of stickers with ECOSYSTEM scope, they could also offer customizable collections of means of expression that users can take to any of their apps, such as photo decoration tools or audio-message recorders. Moreover, future work could explore other mechanisms to provide universal access to user-owned means of expression. For example, inspired in how participants built collections of GIFs and images on their phone's media albums to send them via any app, mobile operating systems could facilitate centralized collections of stickers and other means of expression which apps can then import automatically.

\section{LIMITATIONS AND FUTURE WORK}

Most recruited participants reported having backgrounds related to interface design or software engineering, so the interviews describe practices and problems of users who are both savvy and extreme. We do not claim that our findings apply to more general populations; however, our data shows that participants' drivers for customizing their communication apps came from expression and relationship maintenance needs, which may be relevant to people with more diverse backgrounds. Future work could assess the frequency and distribution of existing customization practices in a broader population as well assess whether some of the customization behaviours we observed with our extreme users, once demonstrated, might be valuable to people in the broader population.

We believe that the insights from this study may become increasingly relevant as people adopt more communication apps [14] and customizations become more popular. For example, searching "stickers for WhatsApp" on the Google Play store results in 1200 apps for adding sticker packs or creating custom ones, all published since WhatsApp incorporated support for stickers in November 2018. Searching for "custom keyboard" results in over 500 third-party soft keyboards with custom backgrounds, stickers and text-input functionality. Moreover, Snapchat has recently integrated their Bitmoji stickers with other social apps such as Tinder ${ }^{4}$ and Houseparty ${ }^{5}$, leveraging the popularity of these customizable stickers to compete in the communication app market [7].

\footnotetext{
${ }^{4}$ Tinder (https://tinder.com) is a location-based social app usually used as a dating platform.

${ }^{5}$ Houseparty (https://houseparty.com/) is a group video chat app.
} 
We focused on studying the breath of customization practices across multiple apps in both iOS and Android to understand their impact in mobile communication. Future work could investigate whether and how users transfer habits of expression between mobile and desktop platforms, or even between mediated and face-to-face communication, e.g., enacting GIFs or mimicking emojis with hand and facial gestures.

\section{CONCLUSIONS}

We studied customization practices in ecosystems of communication apps to understand the value of customizations in the context of CMC and to gather insights that inform novel designs for increased personalization of mobile communication. Through interviews with 15 "extreme users" of communication apps, we collected stories about 22 customization options across nine different apps (e.g., changing the color of chat bubbles in Messenger). We created a taxonomy of customization options in mobile communication apps, which we used to characterize the reported customizations according to their Visibility (whether their results are visible to others or not), Ownership (whether they can be controlled by the user or by other contacts as well) and Scope (whether they affect a single conversation, a whole app, or any conversation throughout the ecosystem of apps).

We found that participants tailored their apps to better express their identities, their organizational culture, and their intimate bonds with others. Participants relied on customization options (e.g., chatbots, custom-made stickers) and means of expression (e.g., emojis, reactions) of particular apps to shape personal forms of expression, which they tried transferring to other apps for maintaining consistent expressions of their identities and bonds with others. However, this was often not possible, leading to expression breakdowns: frustrated attempts to transferring personal forms of expression between apps, mainly due to app-exclusive customization options, customization options with different designs across apps, and app-exclusive means of expression that, despite not being customizable, helped establishing personal forms of expression. To overcome these breakdowns, participants looked for alternative forms of expression within the current app, moved a conversation to the app that best suited their expression needs, or temporarily switched to other apps to generate and export the content they needed for a conversation.

We contribute: 1) a taxonomy of customization options in mobile communication apps that serves as an analytic lens for understanding the role of a customization option in interpersonal communication, as well as a design space for exploring novel opportunities for design; 2) insight into the value of customizations as means for maintaining relationships online; 3 ) insight into "expression breakdowns" and the transfer of habits of expression between apps; 4) implications for designing new customization opportunities for supporting richer expressions of identity and intimacy within close relationships in ecosystems of communication apps.

\section{ACKNOWLEDGMENTS}

This work was partially funded by European Research Council (ERC) grant n³21135 CREATIV: Creating Co-Adaptive Human-Computer Partnerships, and the International Research Fellow Program of the Japan Society for the Promotion of Science (JSPS). We thank all participants for their time and enthusiasm. We also thank the reviewers for their detailed and constructive feedback, as well as the following people for insightful comments and discussions that greatly improved this work: Cliff Lampe, John Tang, Louise Barkhuus, Myriam Lewkowicz, Philip Tchernavskij 凡, Midas Nouwens $@$, Nolwenn Maudet, Germán Leiva, Miguel Renom, Marianela Ciolfi Felice $\bullet$, Ellen Sigloch, Viktor Gustafsson, Elizabeth Walton, Wanyu "Abby" Liu, Alexander Eiselmayer, Diana Lipcanu , Hendrik Heuer , Kengo Shibata and Koji Yatani. 


\section{A REPORTED CUSTOMIZATION OPTIONS}

\begin{tabular}{|c|c|c|c|c|c|c|c|c|}
\hline \multirow{2}{*}{ Customization option } & \multirow{2}{*}{ Reported apps } & \multicolumn{2}{|c|}{ Visibility } & \multicolumn{2}{|c|}{ Ownership } & \multicolumn{3}{|c|}{ Scope } \\
\hline & & Private & Social & Individual & Shared & App & Conversation & Ecosystem \\
\hline \multirow[t]{3}{*}{ Add a custom emoji } & Slack & & $\mathrm{x}$ & & $x$ & $x$ & & \\
\hline & Flowdock & & $\mathrm{x}$ & & $x$ & $x$ & & \\
\hline & HipChat & & $\mathrm{x}$ & & $x$ & $x$ & & \\
\hline \multirow[t]{2}{*}{ Add a custom sticker } & WeChat & & $\mathrm{x}$ & $x$ & & $x$ & & \\
\hline & WhatsApp & & $\mathrm{x}$ & $x$ & & $x$ & & \\
\hline \multirow[t]{2}{*}{ Add a sticker pack } & Messenger & & $x$ & $x$ & & $x$ & & \\
\hline & Telegram & & $\mathrm{x}$ & $\mathrm{x}$ & & $x$ & & \\
\hline Add a custom sticker pack & Telegram & & $\mathrm{x}$ & $\mathrm{x}$ & & $x$ & & \\
\hline \multirow{3}{*}{$\begin{array}{l}\text { Change conversation } \\
\text { background }\end{array}$} & WeChat & $\mathrm{x}$ & & $x$ & & $x$ & $x$ & \\
\hline & Telegram & $x$ & & $\mathrm{x}$ & & $x$ & & \\
\hline & WhatsApp & $x$ & & $x$ & & $x$ & & \\
\hline Change color of chat bubbles & Messenger & & $\mathrm{x}$ & & $x$ & & $x$ & \\
\hline $\begin{array}{l}\text { Change color of chat bubbles } \\
\text { and background (color theme) }\end{array}$ & Telegram & $x$ & & $x$ & & $x$ & & \\
\hline Change emoji shortcut & Messenger & & $\mathrm{x}$ & & $x$ & & $x$ & \\
\hline \multirow[t]{5}{*}{ Change group chat title } & Whatsapp & & $\mathrm{x}$ & & $x$ & & $x$ & \\
\hline & Messenger & & $\mathrm{x}$ & & $x$ & & $x$ & \\
\hline & WeChat & & $x$ & & $x$ & & $x$ & \\
\hline & Telegram & & $\mathrm{x}$ & & $x$ & & $x$ & \\
\hline & Instagram & & $\mathrm{x}$ & & $x$ & & $x$ & \\
\hline Change group chat picture & Whatsapp & & $\mathrm{x}$ & & $x$ & & $x$ & \\
\hline \multirow{3}{*}{$\begin{array}{l}\text { Change contact } \\
\text { nickname }\end{array}$} & Messenger & & $\mathrm{x}$ & & $x$ & & $x$ & \\
\hline & Snapchat & $x$ & & $x$ & & $x$ & & \\
\hline & WeChat & $x$ & & $\mathrm{x}$ & & $x$ & & \\
\hline Change own nickname & Slack & & $\mathrm{x}$ & $\mathrm{x}$ & & $x$ & & \\
\hline Change contact profile picture & $\begin{array}{l}\text { Whatsapp } \\
\text { (discontinued) }\end{array}$ & $x$ & & $x$ & & $x$ & & \\
\hline \multirow[t]{2}{*}{ Set favorite contacts } & WeChat & $x$ & & $x$ & & $x$ & & \\
\hline & Telegram & $x$ & & $\mathrm{x}$ & & $x$ & & \\
\hline \multirow[t]{3}{*}{ Change notification sound } & Whatsapp & $x$ & & $x$ & & $x$ & $x$ & \\
\hline & Telegram & $x$ & & $\mathrm{x}$ & & $x$ & $x$ & \\
\hline & Messenger & $x$ & & $x$ & & $x$ & & \\
\hline \multirow[t]{2}{*}{ Add chatbot } & Slack & & $\mathrm{x}$ & & $x$ & $x$ & $x$ & \\
\hline & Telegram & & $\mathrm{x}$ & $\mathrm{x}$ & $x$ & $x$ & $x$ & \\
\hline \multirow[t]{2}{*}{ Add custom chatbot } & Slack & & $\mathrm{x}$ & & $x$ & $x$ & $x$ & \\
\hline & Telegram & & $\mathrm{x}$ & $\mathrm{x}$ & $x$ & $x$ & $x$ & \\
\hline Change Slackbot settings & Slack & & $\mathrm{x}$ & & $x$ & $x$ & & \\
\hline Change status message & Slack & & $\mathrm{x}$ & $x$ & & $x$ & & \\
\hline \multirow[t]{2}{*}{ Install plugin (e.g. polls, games) } & Messenger & & $x$ & $x$ & & $x$ & & \\
\hline & WeChat & & $\mathrm{x}$ & $\mathrm{x}$ & & $x$ & & \\
\hline \multirow[t]{2}{*}{ Add alternative soft-keyboard } & Bitmoji Keyboard & & $\mathrm{x}$ & $\mathrm{x}$ & & & & $x$ \\
\hline & $\begin{array}{l}\text { Tenor Keyboard (for } \\
\text { searching GIFs) }\end{array}$ & & $x$ & $x$ & & & & $x$ \\
\hline Add stickers to soft-keyboard & Gboard & & $\mathrm{x}$ & $x$ & & & & $x$ \\
\hline
\end{tabular}

Fig. 4. List of reported customizations options in mobile communication apps characterized by their type of Visibility, Ownership and Scope. Grey cells highlight differences across apps. The table only shows apps and customization options mentioned in the interviews.

\section{REFERENCES}

[1] Mark S. Ackerman. 2000. The Intellectual Challenge of CSCW: The Gap Between Social Requirements and Technical Feasibility. Hum.-Comput. Interact. 15, 2 (Sept. 2000), 179-203. https://doi.org/10.1207/S15327051HCI1523_5

[2] Saeideh Bakhshi, David A. Shamma, Lyndon Kennedy, Yale Song, Paloma de Juan, and Joseph 'Jofish' Kaye. 2016. Fast, Cheap, and Good: Why Animated GIFs Engage Us. ACM Press, 575-586. https://doi.org/10.1145/2858036.2858532 
[3] Elizabeth Bales, Kevin A. Li, and William Griwsold. 2011. CoupleVIBE: Mobile Implicit Communication to Improve Awareness for (Long-distance) Couples. In Proceedings of the ACM 2011 Conference on Computer Supported Cooperative Work (CSCW'11). ACM, New York, NY, USA, 65-74. https://doi.org/10.1145/1958824.1958835

[4] Virginia Braun and Victoria Clarke. 2006. Using thematic analysis in psychology. Qualitative research in psychology 3 , 2 (2006), 77-101.

[5] Andrea Bunt, Cristina Conati, and Joanna McGrenere. 2007. Supporting interface customization using a mixed-initiative approach. In Proceedings of the 12th international conference on Intelligent user interfaces. ACM, 92-101.

[6] Eun Kyoung Choe, Nicole B. Lee, Bongshin Lee, Wanda Pratt, and Julie A. Kientz. 2014. Understanding Quantifiedselfers' Practices in Collecting and Exploring Personal Data. In Proceedings of the SIGCHI Conference on Human Factors in Computing Systems (CHI '14). ACM, New York, NY, USA, 1143-1152. https://doi.org/10.1145/2556288.2557372

[7] Josh Constine. 2019. To stop copycats, Snapchat shares itself. TechCrunch (Apr 2019). https://techcrunch.com/2019/ 04/07/rise-of-the-snapchat-empire/

[8] Henriette Cramer, Paloma de Juan, and Joel Tetreault. 2016. Sender-intended functions of emojis in US messaging. ACM Press, 504-509. https://doi.org/10.1145/2935334.2935370

[9] Henriette Cramer and Maia L. Jacobs. 2015. Couples' Communication Channels: What, When \& Why? In Proceedings of the 33rd Annual ACM Conference on Human Factors in Computing Systems (CHI '15). ACM, New York, NY, USA, 709-712. https://doi.org/10.1145/2702123.2702356

[10] Michael A. DeVito, Jeremy Birnholtz, and Jeffery T. Hancock. 2017. Platforms, People, and Perception: Using Affordances to Understand Self-Presentation on Social Media. In Proceedings of the 2017 ACM Conference on Computer Supported Cooperative Work and Social Computing (CSCW '17). ACM, New York, NY, USA, 740-754. https://doi.org/10.1145/ 2998181.2998192

[11] Michael A. DeVito, Jeremy Birnholtz, Jeffery T. Hancock, Megan French, and Sunny Liu. 2018. How People Form Folk Theories of Social Media Feeds and What It Means for How We Study Self-Presentation. In Proceedings of the 2018 CHI Conference on Human Factors in Computing Systems (CHI '18). ACM, New York, NY, USA, Article 120, 12 pages. https://doi.org/10.1145/3173574.3173694

[12] Michael A. DeVito, Ashley Marie Walker, and Jeremy Birnholtz. 2018. 'Too Gay for Facebook': Presenting LGBTQ+ Identity Throughout the Personal Social Media Ecosystem. Proc. ACM Hum.-Comput. Interact. 2, CSCW, Article 44 (Nov. 2018), 23 pages. https://doi.org/10.1145/3274313

[13] John Partomo Djajadiningrat, William W Gaver, and JW Fres. 2000. Interaction relabelling and extreme characters: methods for exploring aesthetic interactions. In Proceedings of the 3rd conference on Designing interactive systems: processes, practices, methods, and techniques. ACM, 66-71.

[14] Maeve Duggan and Nicole B Ellison. 2015. Social media update 2014. (2015).

[15] Jeff Dyck, David Pinelle, Barry AT Brown, and Carl Gutwin. 2003. Learning from Games: HCI Design Innovations in Entertainment Software.. In Graphics interface, Vol. 2003. Citeseer, 237-246.

[16] Leah Findlater and Joanna McGrenere. 2004. A comparison of static, adaptive, and adaptable menus. In Proceedings of the SIGCHI conference on Human factors in computing systems. ACM, 89-96.

[17] Leah Findlater, Joanna McGrenere, and David Modjeska. 2008. Evaluation of a role-based approach for customizing a complex development environment. In Proceedings of the SIGCHI Conference on Human Factors in Computing Systems. ACM, 1267-1270.

[18] Erving Goffman. 1959. The Presentation of Self in. Butler, Bodies that Matter (1959).

[19] Carla F. Griggio, Midas Nouwens, Joanna McGrenere, and Wendy E. Mackay. 2019. Augmenting Couples' Communication with Lifelines: Shared Timelines of Mixed Contextual Information. In Proceedings of the 2019 CHI Conference on Human Factors in Computing Systems (CHI '19). ACM, New York, NY, USA, Article 623, 13 pages. https://doi.org/10.1145/3290605.3300853

[20] Marc Hassenzahl, Stephanie Heidecker, Kai Eckoldt, Sarah Diefenbach, and Uwe Hillmann. 2012. All You Need is Love: Current Strategies of Mediating Intimate Relationships Through Technology. ACM Trans. Comput.-Hum. Interact. 19, 4 , Article 30 (Dec. 2012), 19 pages. https://doi.org/10.1145/2395131.2395137

[21] Mariam Hassib, Daniel Buschek, Pawel W. Wozniak, and Florian Alt. 2017. HeartChat: Heart Rate Augmented Mobile Chat to Support Empathy and Awareness. In Proceedings of the 2017 CHI Conference on Human Factors in Computing Systems (CHI '17). ACM, New York, NY, USA, 2239-2251. https://doi.org/10.1145/3025453.3025758

[22] Caroline Haythornthwaite. 2005. Social networks and Internet connectivity effects. Information, Community \& Society 8, 2 (2005), 125-147.

[23] Jialun Jiang, Casey Fiesler, and Jed R Brubaker. 2018. 'The Perfect One': Understanding Communication Practices and Challenges with Animated GIFs. Proceedings of the ACM on Human-Computer Interaction 2, CSCW (2018), 80.

[24] Helge Kahler. 2001. More Than WORDs - Collaborative Tailoring of a Word Processor. fournal of Universal Computer Science 7, 8 (2001), 826-847. 
[25] Ryan Kelly, Daniel Gooch, Bhagyashree Patil, and Leon Watts. 2017. Demanding by design: Supporting effortful communication practices in close personal relationships. In Proceedings of the 2017 ACM Conference on Computer Supported Cooperative Work and Social Computing. ACM, 70-83.

[26] Ryan Kelly, Daniel Gooch, and Leon Watts. 2018. 'It's More Like a Letter': An Exploration of Mediated Conversational Effort in Message Builder. Proceedings of the ACM on Human-Computer Interaction 2, CSCW (2018), 87.

[27] Ryan Kelly and Leon Watts. 2015. Characterising the inventive appropriation of emoji as relationally meaningful in mediated close personal relationships. Experiences of Technology Appropriation: Unanticipated Users, Usage, Circumstances, and Design (2015).

[28] Lorenz Cuno Klopfenstein, Saverio Delpriori, Silvia Malatini, and Alessandro Bogliolo. 2017. The rise of bots: A survey of conversational interfaces, patterns, and paradigms. In Proceedings of the 2017 Conference on Designing Interactive Systems. ACM, 555-565.

[29] Joon Young Lee, Nahi Hong, Soomin Kim, Jonghwan Oh, and Joonhwan Lee. 2016. Smiley face: why we use emoticon stickers in mobile messaging. ACM Press, 760-766. https://doi.org/10.1145/2957265.2961858

[30] Christian Licoppe. 2004. 'Connected' presence: The emergence of a new repertoire for managing social relationships in a changing communication technoscape. Environment and planning D: Society and space 22, 1 (2004), 135-156.

[31] Wendy E Mackay. 1990. Users and customizable software: A co-adaptive phenomenon. Ph.D. Dissertation. Citeseer.

[32] Wendy E Mackay. 1991. Triggers and barriers to customizing software. In Proceedings of the SIGCHI conference on Human factors in computing systems. ACM, 153-160.

[33] Wendy E Mackay. 2000. Responding to cognitive overload: Co-adaptation between users and technology. Intellectica 30, 1 (2000), 177-193.

[34] Joanna McGrenere, Ronald M Baecker, and Kellogg S Booth. 2007. A field evaluation of an adaptable two-interface design for feature-rich software. ACM Transactions on Computer-Human Interaction (TOCHI) 14, 1 (2007), 3.

[35] Sarah McRoberts, Haiwei Ma, Andrew Hall, and Svetlana Yarosh. 2017. Share First, Save Later: Performance of Self through Snapchat Stories. ACM Press, 6902-6911. https://doi.org/10.1145/3025453.3025771

[36] Hannah Miller, Jacob Thebault-Spieker, Shuo Chang, Isaac Johnson, Loren Terveen, and Brent Hecht. 2016. Blissfully happy" or "ready to fight": Varying Interpretations of Emoji. Proceedings of ICWSM 2016 (2016)

[37] Hannah Miller Hillberg, Zachary Levonian, Daniel Kluver, Loren Terveen, and Brent Hecht. 2018. What I See is What You Don'T Get: The Effects of (Not) Seeing Emoji Rendering Differences Across Platforms. Proc. ACM Hum.-Comput. Interact. 2, CSCW, Article 124 (Nov. 2018), 24 pages. https://doi.org/10.1145/3274393

[38] Kate M. Miltner and Tim Highfield. 2017. Never gonna GIF you up: Analyzing the cultural significance of the animated GIF. Social Media+ Society 3, 3 (2017), 2056305117725223.

[39] Katie Moon and Deborah Blackman. 2014. A guide to understanding social science research for natural scientists. Conservation Biology 28, 5 (2014), 1167-1177.

[40] Bonnie A. Nardi, Steve Whittaker, and Erin Bradner. 2000. Interaction and Outeraction: Instant Messaging in Action In Proceedings of the 2000 ACM Conference on Computer Supported Cooperative Work (CSCW'00). ACM, New York, NY, USA, 79-88. https://doi.org/10.1145/358916.358975

[41] Midas Nouwens, Carla F Griggio, and Wendy E Mackay. 2017. WhatsApp is for family; Messenger is for friends: Communication Places in App Ecosystems. In Proceedings of the 2017 CHI Conference on Human Factors in Computing Systems. ACM, 727-735.

[42] Kenton P. O'Hara, Michael Massimi, Richard Harper, Simon Rubens, and Jessica Morris. 2014. Everyday Dwelling with WhatsApp. In Proceedings of the 17th ACM Conference on Computer Supported Cooperative Work \& Social Computing (CSCW'14). ACM, New York, NY, USA, 1131-1143. https://doi.org/10.1145/2531602.2531679

[43] Antti Oulasvirta and Jan Blom. 2008. Motivations in Personalisation Behaviour. Interact. Comput. 20, 1 (Jan. 2008), 1-16. https://doi.org/10.1016/j.intcom.2007.06.002

[44] Stanley R Page, Todd J Johnsgard, Uhl Albert, and C Dennis Allen. 1996. User customization of a word processor. In Proceedings of the SIGCHI Conference on Human Factors in Computing Systems. ACM, 340-346.

[45] Jaram Park, Vladimir Barash, Clay Fink, and Meeyoung Cha. 2013. Emoticon Style: Interpreting Differences in Emoticons Across Cultures.. In Icwsm.

[46] Matthias Peissner and Rob Edlin-White. 2013. User control in adaptive user interfaces for accessibility. In IFIP Conference on Human-Computer Interaction. Springer, 623-640.

[47] Lauren E. Scissors and Darren Gergle. 2013. "Back and Forth, Back and Forth": Channel Switching in Romantic Couple Conflict. In Proceedings of the 2013 Conference on Computer Supported Cooperative Work (CSCW'13). ACM, New York, NY, USA, 237-248. https://doi.org/10.1145/2441776.2441804

[48] Manya Sleeper, William Melicher, Hana Habib, Lujo Bauer, Lorrie Faith Cranor, and Michelle L. Mazurek. 2016. Sharing personal content online: Exploring channel choice and multi-channel behaviors. In Proceedings of the 2016 CHI conference on human factors in computing systems. ACM, 101-112.

[49] Aaron Smith and Monica Anderson. 2018. Social media use in 2018. Pew research center (2018). 
[50] Olli Sotamaa. 2010. When the game is not enough: Motivations and practices among computer game modding culture. Games and Culture 5, 3 (2010), 239-255.

[51] Frederic Stutzman, Jessica Vitak, Nicole B Ellison, Rebecca Gray, and Cliff Lampe. 2012. Privacy in Interaction: Exploring Disclosure and Social Capital in Facebook.. In ICWSM.

[52] Satomi Sugiyama. 2015. Kawaii meiru and Maroyaka neko : Mobile emoji for relationship maintenance and aesthetic expressions among Japanese teens. First Monday 20, 10 (2015). https://doi.org/10.5210/fm.v20i10.5826

[53] Aurélien Tabard, Wendy Mackay, Nicolas Roussel, and Catherine Letondal. 2007. Pagelinker: integrating contextual bookmarks within a browser. In Proceedings of the SIGCHI conference on Human factors in computing systems. ACM, 337-346.

[54] Lee Taber and Steve Whittaker. 2018. Personality Depends on The Medium: Differences in Self-Perception on Snapchat, Facebook and Offline. ACM Press, 1-13. https://doi.org/10.1145/3173574.3174181

[55] Garreth W. Tigwell and David R. Flatla. 2016. Oh That's What You Meant!: Reducing Emoji Misunderstanding. In Proceedings of the 18th International Conference on Human-Computer Interaction with Mobile Devices and Services Adjunct (MobileHCI '16). ACM, New York, NY, USA, 859-866. https://doi.org/10.1145/2957265.2961844

[56] Jackson Tolins and Patrawat Samermit. 2016. GIFs as Embodied Enactments in Text-Mediated Conversation. Research on Language and Social Interaction 49, 2 (April 2016), 75-91. https://doi.org/10.1080/08351813.2016.1164391

[57] Emily Troshynski, Charlotte Lee, and Paul Dourish. 2011. Accountabilities of presence: reframing location-based systems. Droit et cultures. Revue internationale interdisciplinaire 61 (2011), 171-193.

[58] Zeynep Tufekci. 2008. Can you see me now? Audience and disclosure regulation in online social network sites. Bulletin of Science, Technology \& Society 28, 1 (2008), 20-36.

[59] Francesco Vitale, Izabelle Janzen, and Joanna McGrenere. 2018. Hoarding and Minimalism: Tendencies in Digital Data Preservation. In Proceedings of the 2018 CHI Conference on Human Factors in Computing Systems (CHI '18). ACM, New York, NY, USA, Article 587, 12 pages. https://doi.org/10.1145/3173574.3174161

[60] Joseph B Walther. 1996. Computer-mediated communication: Impersonal, interpersonal, and hyperpersonal interaction. Communication research 23, 1 (1996), 3-43.

[61] Daricia Wilkinson, Moses Namara, Karla Badillo-Urquiola, Pamela J. Wisniewski, Bart P. Knijnenburg, Xinru Page, Eran Toch, and Jen Romano-Bergstrom. 2018. Moving Beyond a "One-size Fits All": Exploring Individual Differences in Privacy. In Extended Abstracts of the 2018 CHI Conference on Human Factors in Computing Systems (CHI EA '18). ACM, New York, NY, USA, Article W16, 8 pages. https://doi.org/10.1145/3170427.3170617

[62] Terry Winograd and Fernando Flores. 1987. On understanding computers and cognition: A new foundation for design: A response to the reviews. Elsevier.

[63] Sarah Wiseman and Sandy J. J. Gould. 2018. Repurposing Emoji for Personalised Communication: Why Pizza Means "I Love You". In Proceedings of the 2018 CHI Conference on Human Factors in Computing Systems (CHI '18). ACM, New York, NY, USA, Article 152, 10 pages. https://doi.org/10.1145/3173574.3173726

[64] Nick Yee, Nicolas Ducheneaut, Les Nelson, and Peter Likarish. 2011. Introverted elves \& conscientious gnomes: the expression of personality in world of warcraft. In Proceedings of the SIGCHI Conference on Human Factors in Computing Systems. ACM, 753-762.

[65] Amy X Zhang and Justin Cranshaw. 2018. Making sense of group chat through collaborative tagging and summarization. Proceedings of the ACM on Human-Computer Interaction 2, CSCW (2018), 196.

[66] Xuan Zhao, Cliff Lampe, and Nicole B. Ellison. 2016. The Social Media Ecology: User Perceptions, Strategies and Challenges. ACM Press, 89-100. https://doi.org/10.1145/2858036.2858333

[67] Rui Zhou, Jasmine Hentschel, and Neha Kumar. 2017. Goodbye Text, Hello Emoji: Mobile Communication on WeChat in China. ACM Press, 748-759. https://doi.org/10.1145/3025453.3025800

Received April 2019; revised June 2019; accepted August 2019 